\title{
A IMPORTÂNCIA DO ATENDIMENTO PSICOSSOCIAL DO NASF COMO IGUALDADE DE ACESSO À SAÚDE PÚBLICA BRASILEIRA: INCLUSÃO DA PESSOA IDOSA
}

\section{ARTIGO ORIGINAL}

GEHM, Maria Madalena Marques ${ }^{1}$

BRUMATI, Lêda Cristiane dos Santos ${ }^{2}$

MARTINS, Nathiele da Silva ${ }^{3}$

GEHM, Maria Madalena Marques. BRUMATI, Lêda Cristiane dos Santos. MARTINS, Nathiele da Silva. A importância do atendimento psicossocial do NASF como igualdade de acesso à saúde pública brasileira: Inclusão da pessoa idosa. Revista Científica Multidisciplinar Núcleo do Conhecimento. Ano 04, Ed. 12, Vol. 02, pp. 05-13. Dezembro de 2019. ISSN: 2448-0959, Link de acesso: https://www.nucleodoconhecimento.com.br/psicologia/atendimentopsicossocial

\footnotetext{
${ }^{1}$ Mestre em Extensão Rural pela Universidade Federal de Santa Maria- RS; Bacharel em Serviço Social pela Unicruz.

${ }^{2}$ Especialista em: Gestão da Educação Pública pela Universidade Federal de Mato Grosso; Psicopedagogia Institucional pela Educon-Sociedade de Educação Continuada; Educação a Distância, Tutoria, Metodologia e Aprendizagem pela Educon-Sociedade de Educação Continuada; Educação Infantil e Séries Iniciais pela Educon-Sociedade de Educação Continuada; Licenciada em Pedagogia pela Universidade Federal de Mato Grosso; Bacharel em Serviço Social pelo Centro Universitário da Grande Dourados/MS.

${ }^{3}$ Especialista Gestão da Saúde - Instituto Prominas; Graduada em Psicologia pelo Centro Universitário da Grande Dourados/MS.
} 


\section{RESUMO}

O presente artigo ressalta o tema: $\mathrm{O}$ atendimento psicossocial do Nasf como igualdade de acesso à saúde pública brasileira: Inclusão da pessoa idosa, e teve como objetivo considerar que o atendimento psicossocial na área da saúde pública brasileira oportuniza igualdade de acesso aos serviços disponíveis para o cidadão que se encontra em estado vulnerável, buscando atendimento de qualidade com vista a possibilitar seu tratamento clínico com maior agilidade, não permitindo que os entraves do sistema o excluam socialmente. Os dados foram levantados e coletados por meio de pesquisas bibliográficas sobre o tema com o intuito de dar objetividade, ou seja, dar um raciocínio conclusivo sobre o tema da pesquisa por intermédio de análise documental sistemática das argumentações teóricas pesquisadas. Por meio da análise, os dados obtidos sugeriram que 0 atendimento psicossocial a pessoa idosa na saúde pública brasileira é um instrumento de combate à exclusão social do indivíduo e contribui para possibilitar acesso integral aos serviços de atendimento.

Palavras-chave: Idoso, igualdade, inclusão, saúde.

\section{INTRODUÇÃO}

O presente trabalho tem como tema 0 atendimento psicossocial do Nasf como igualdade de acesso à saúde pública brasileira: inclusão da pessoa idosa, o qual requer como todo ser humano, atenção e respeito por tudo que já foram e porque um dia também nos tornaremos idosos e com certeza gostaríamos de ser tratados com dignidade.

Nesta perspectiva, construiu-se questões que nortearam este trabalho:

- Qual a relevância do Núcleo de Apoio a Saúde da Família na saúde pública?

- De que forma o atendimento psicossocial do Núcleo de Apoio a Saúde da Família contribui na atenção ao idoso?

A população mundial está envelhecendo, principalmente em países em desenvolvimento como o Brasil, o que se torna um desafio para o Sistema Único de 
Saúde (SUS), pois a atenção à saúde do idoso reveste-se de grande preocupação, visto que essa faixa etária apresenta necessidades específicas caracterizadas pela cronicidade e complexidade, interferindo bruscamente na qualidade de vida o que demanda cuidados adequados.

Além dos determinantes econômicos do cuidado à saúde do idoso, é importante investigar a natureza e qualidade do cuidado prestado. Por isso, ao optar por este tema buscou-se, como objetivo primordial, pesquisar através de diversas bibliografias a importância do atendimento psicossocial como ferramenta que possibilita a pessoa idosa maior atenção no atendimento prioritário à saúde. Portanto, utilizou-se como recurso metodológico a pesquisa bibliográfica, realizada a partir da análise de materiais publicados na literatura e artigos científicos divulgados no meio eletrônico.

O texto final foi fundamentado nas ideias e concepções de autores como: Caldas (2011), Filho (2008), Moraes (2012), Veras (2001) e Vasconcelos (2012).

\subsection{CONTEXTUALIZANDO NASF}

O NASF - Núcleo de Apoio a Saúde da Família foi criado pelo Ministério da Saúde em 2008 com o objetivo de apoiar a consolidação da Atenção Básica no Brasil, ampliando as ofertas de saúde na rede de serviços, assim como a resolutividade, a abrangência e o alvo das ações. É uma equipe formada por diversos profissionais de áreas distintas (conhecimento), que atuam de forma totalmente integrada cujas atuações visam apoiar os profissionais das Equipes de Saúde da Família, das Equipes de Atenção Básica, direcionando suas ações para populações específicas, por meio do compartilhamento de práticas e saberes em saúde, baseado nos princípios da integralidade e da interdisciplinaridade, o que o diferencia de tantos outros programas já implantados que não tiveram tanta eficácia , haja visto que possuíam o mesmo foco, mas sem a mesma resolutividade, onde no Nasf a proposta é mais prevenção do que clínica ampliada.

No âmbito da jurisprudência, a regulamentação do Nasf de acordo com Vasconcelos (2012, p. 73) se dá: 
Pela Portaria no 2.488, de 21 de outubro de 2011, onde se configuram como equipes multiprofissionais que atuam de forma integrada com as equipes de Saúde da Família (eSF), as equipes de atenção básica para populações específicas (consultórios na rua, equipes ribeirinhas e fluviais) e com o Programa Academia da Saúde. Esta atuação integrada permite realizar discussões de casos clínicos, possibilita o atendimento compartilhado entre profissionais tanto na Unidade de Saúde como nas visitas domiciliares, permite a construção conjunta de projetos terapêuticos de forma que amplia e qualifica as intervenções no território e na saúde de grupos populacionais. Essas ações de saúde também podem ser intersetoriais, com foco prioritário nas ações de prevenção e promoção da saúde. Há que se considerar ainda que existe repasses de recursos (implantação e custeio) do Governo Federal para o programa.

Tais ações são realizadas dentro de sua territorialidade, objetivando ampliar as ações da atenção básica e também contribuir para a integralidade do cuidado aos usuários do SUS - Sistema Único de Saúde, principalmente por intermédio da ampliação da clínica, auxiliando no aumento da capacidade de análise e de intervenção sobre problemas e necessidades de saúde, tanto em termos clínicos quanto sanitários e ambientais dentro dos territórios, priorizando intervenções coletivas de promoção e acompanhamento de grupos sociais em vulnerabilidade, e desta forma com atendimentos voltados para com a prevenção se contribua para aumentar a resolutividade da própria atenção básica.

Podemos citar ainda que o Nasf, por intermédio multidisciplinar de sua equipe desenvolvem ações de apoio matricial nas discussão de casos, atendimentos compartilhados (NASF + ESF vinculada), atendimentos individuais precedida ou seguida de discussão com a ESF, construção conjunta de projetos terapêuticos, ações de educação permanente, intervenções no território e na saúde de grupos populacionais e da coletividade, ações intersetoriais, ações de prevenção e promoção da saúde, discussão do processo de trabalho das equipes dentre outros. (BRASIL,2010).

A multidisciplinaridade desses profissionais tem por prioridade buscar a efetividade de suas ações de forma compartilhada atuando de forma gerencial a atender as demandas na saúde e suas particularidades no que se refere a Atenção Básica a saúde, visando combater toda e qualquer forma de exclusão e falta de acesso a uma 
saúde pública de qualidade, cujas atividades fomentam ainda fortalecer as ações de promoção de saúde, estabelecimento e fortalecimento de vínculos, além dos estímulo a autonomia e protagonismo, principalmente no ciclo de vida dos idosos.

\subsection{IGUALDADE DE ACESSO À SAÚDE PÚBLICA: BREVE RELATO}

Segundo a Organização Mundial de Saúde (OMS) o termo "saúde" é definido como um completo estado de bem estar físico, mental e social, sem consistir apenas na ausência de enfermidade ou doença. Em conformidade com a Constituição Federal do Brasil (1988) menciona que a saúde é um direito de todos e fundamental para garantir o acesso universal e igualitário de forma integral ao indivíduo, sendo um direito social (art.6ํ) de natureza pública, tendo em vista que se exige uma prestação do Estado e da Sociedade no que se refere a inserção de políticas públicas que assegure a plenitude de acesso e tratamento integral à saúde, onde os entes federados (União, Estados, Distrito Federal e Municípios) têm a responsabilidade de organizar e instituir essas políticas públicas da saúde para com o bom atendimento da população (art. 23,II), através também de redirecionamento de recursos para tal.

Para que se efetive de fato o acesso igualitário e universal à saúde como preconiza a Carta Magna Brasileira, este deve ser compreendido de forma a assegurar a universalidade das pessoas com ampla cobertura de atendimento aos serviços de saúde ofertados, visando reduzir o risco de doenças e oportunizar tratamento para quem necessita de recuperação, compreendendo assim ações e serviços que priorizem a promoção, proteção e recuperação (art. 196), cuja cobertura dos serviços se realize de modo a permitir atendimento à todos dentro do contexto da equidade, em sendo é a igualdade de assistência à saúde, sem preconceitos ou privilégios de qualquer espécie, e assim neste contexto se incorpora o parâmetro de justiça à distribuição igualitária de acesso à saúde pública.

A cronicidade das doenças mais prevalentes entre os idosos deve ser levada em consideração quando da organização dos serviços de saúde. Acrescente-se a este fato que os problemas de saúde dos idosos, além de serem de longa duração, exigem pessoal qualificado, equipe 
multidisciplinar, equipamentos e exames complexos e de alto custo. (VERAS, 2001, p.23-27)

Desta forma a atuação do Nasf frente às necessidades da pessoa idosa, fomenta ações de proteção à saúde da pessoa idosa via igualdade de acesso se dá em virtude do princípio da assistência, as pessoas idosas e estas devem ter acesso a cuidados de saúde tanto física, quanto mental e emocional e que previnam ou atrasem o possível aparecimento de doenças, as ajudando a conservar ou a readquirir um nível ótimo de bem-estar.

A competência do Estado em garantir à pessoa idosa que sua saúde seja protegida precisa ser respeitada e concretizada de forma contínua, e isso só ocorre via mecanismo de efetivação de políticas sociais públicas. Tais políticas permitem que as pessoas tenham um envelhecimento saudável e digno. Dentre a legislação pertinente ao caso, podemos citar que a maior parte das leis internacionais de direitos humanos tem colocado o direito a saúde como um direito social subjetivo das pessoas (FILHO, 2008).

\subsection{POLÍTICAS PARA O RESGUARDO DA SAÚDE IDOSO NO BRASIL}

A Organização Mundial da Saúde determina as bases para um envelhecimento saudável, sobressaindo a equidade no acesso aos cuidados de saúde e o desenvolvimento continuado de ações de promoção à saúde e prevenção de doenças. No que tange as políticas criadas para que tal definição seja cumprida podemos citar a Política Nacional de Saúde do Idoso (PNSI), criada através da Portaria no 1395/1999, do Ministério da Saúde (MS), visou à promoção do envelhecimento saudável, à prevenção de doenças, à recuperação da saúde, à preservação/melhoria/reabilitação da capacidade funcional dos idosos com a finalidade de assegurar-Ihes sua permanência no meio e sociedade em que vivem, de forma que possam desempenhar suas atividades de maneira independente.

É importante destacar ainda que nesta política estão elencadas diversas diretrizes que enfatizam a promoção do envelhecimento saudável sendo parâmetros que auxiliam na efetivação deste atendimento em sendo: 
- Envelhecimento ativo e saudável;

- Atenção integral e integrada à saúde da pessoa idosa;

- Estímulo às ações intersetoriais;

- Fortalecimento do controle social, dentre outras.

Tais diretrizes tem por objetivo orientar a organização do cuidado ofertado à pessoa idosa no âmbito do SUS, potencializando as ações já desenvolvidas e propondo estratégias para fortalecer a articulação, a qualificação do cuidado e a ampliação do acesso da pessoa idosa aos pontos de atenção das Redes de Atenção à Saúde.

O desafio é ampliar o acesso, incluir e/ou potencializar o cuidado integral, concretizar ações intersetoriais nos territórios com foco nas especificidades e demandas de cuidado da população idosa. Voltado ao desenvolvimento de ações que orientem a melhoria de suas habilidades funcionais, mediante a adoção precoce de hábitos saudáveis de vida, a eliminação de comportamentos nocivos à saúde, bem como destaca ainda todas as ações no setor saúde e as responsabilidades institucionais de cada ente federado na promoção desta.

Compete destacar ainda o Estatuto do Idoso outro dispositivo que norteia ações sociais e de saúde, visando garantir os direitos das pessoas idosas e a obrigação do Estado à proteção dos mesmos.

É sabido que o envelhecimento nos coloca frente a inúmeros desafios, dentre eles a produzir políticas de saúde que venham atender às necessidades das pessoas idosas, levando em consideração que a proporção de usuários idosos de todos os serviços prestados tende a ser cada vez maior, quer pelo maior acesso às informações do referido grupo etário, quer pelo seu expressivo aumento relativo e absoluto na população brasileira, via de regra tem um uma população expressiva nessa faixa etária.

Neste contexto é importante destacar que de acordo com Camacho (2010, p. 107)

A velhice não necessita da totalidade de sua reserva funcional para viver bem e com qualidade e não deve ser considerada como doença, pois as 
enfermidades mais comuns nessa etapa da vida são preveníveis, diagnosticáveis e tratáveis. À medida que as tendências demográficas aceleram, há um aumento na prevalência de doenças crônicas, o que implica a urgência de priorizar a prevenção em todos os países, no entanto, apesar de preconizar suas prioridades para atenção básica, o SUS é orientado pelo imediatismo, com ênfase no cuidado agudo, e não na prevenção e no cuidado crônico.

Assim, no que tange a questão de instrumentos legais quanto ao cuidado do idoso pode-se mencionar que estes tem por objetivo permitir um envelhecimento saudável, o que significa preservar sua capacidade funcional, sua autonomia e manter o nível de qualidade de vida, em consonância com os princípios e diretrizes do Sistema Único de Saúde (SUS), que direcionam medidas individuais e coletivas em todos os níveis de atenção à saúde.

\subsection{O ATENDIMENTO PSICOSSOCIAL AO IDOSO: DIREITO À SAÚDE}

Envelhecer é um fenômeno que diz respeito a todos nós. Vários autores conceituam o termo velhice, mas de acordo com o estabelecido na Política Nacional do Idoso (PNI), Lei no 8.842, de 04 de janeiro de 1994, considera-se como tal, a população com 60 anos ou mais. Concordando com Moraes (2012), a velhice é uma etapa da vida com características e valores próprios, em que ocorrem modificações no indivíduo, tanto na estrutura orgânica, como no metabolismo, no equilíbrio bioquímico, na imunidade, na nutrição, nos mecanismos funcionais, nas características intelectuais e emocionais.

Neste contexto podemos citar que:

Como o envelhecimento não é um processo homogêneo, necessidades e demandas dos idosos variam, sendo preciso fortalecer o trabalho em rede para contemplar a atenção aos idosos saudáveis e atender àqueles com diferentes graus de incapacidade ou enfermidade, inclusive nos domicílios. Assim, o adequado cuidado ao idoso demanda um sistema de saúde coordenado, com cada instância contribuindo para as ações das demais. (CALDAS, 2011, p.87).

Partindo desse princípio através do atendimento psicossocial busca-se incluir a pessoa idosa em programas que priorizem de forma ímpar seu atendimento aos 
programas de saúde que tem por finalidade favorecer a pessoa idosa no que tange a atenção de seus direitos na área da saúde por intermédio de ações implementadoras de políticas públicas de saúde para atendimento aos mesmos.

Partindo desse pressuposto, o atendimento psicossocial na saúde através da equipe do Nasf tem como intuito oportunizar atender e orientar a pessoa idosa quanto a sua inclusão aos serviços de atendimento prioritário a saúde, inclusão em participação de atividades grupais que visam qualidade de vida.

O atendimento psicossocial a pessoa idosa na saúde fomenta ações que priorizam que esta clientela tenha seus direitos salvaguardados através de atendimento prioritário e inclusivo nas políticas públicas voltadas para contemplar essa fase de vida, levando em consideração que o envelhecimento é uma experiência heterogênea.

Um grande aliado para concretização dessas políticas é o Estatuto do Idoso (Lei Federal 10.741/2003) que elenca a regulamentação dos direitos dos cidadãos com mais de sessenta anos, onde preza a observância dos direitos da pessoa idosa, ligadas às deliberações mais específicas no âmbito da saúde do idoso, a Lei citada, visa:

Garantir ao idoso a assistência à saúde, nos diversos níveis de atendimento do Sistema Único de Saúde; Prevenir, promover, proteger e recuperar a saúde do idoso, mediante programas e medidas profiláticas; Adotar e aplicar normas de funcionamento às instituições geriátricas e similares, com fiscalização pelos gestores do Sistema Único de Saúde; Elaborar normas de serviços geriátricos hospitalares; Desenvolver formas de cooperação entre as Secretarias de Saúde dos Estados, do Distrito Federal, e dos Municípios e entre os Centros de Referência em Geriatria e Gerontologia para treinamento de equipes interprofissionais; Incluir a Geriatria como especialidade clínica, para efeito de concursos públicos federais, estaduais, do Distrito Federal e municipais; Realizar estudos para detectar o caráter epidemiológico de determinadas doenças do idoso, com vistas a prevenção, tratamento e reabilitação; e criar serviços alternativos de saúde para o idoso (BRASIL, 2010, p.17).

Ante o exposto é imprescindível destacar que a desenvoltura da equipe multidisciplinar na inclusão da pessoa idosa no atendimento igualitária na saúde 
pública brasileira busca diminuir as desigualdades sociais que acarretam nosso sistema público de saúde. O grande desafio das políticas públicas de saúde dos idosos é sem sombra de dúvidas, adotar e manter programas de promoção e proteção da saúde e prevenção de doenças.

\section{CONSIDERAÇÕES FINAIS}

Em virtude da observância realizada, foi possível averiguar que é grande demanda de acompanhamento de casos que envolvem a atenção à pessoa idosa, onde se busca os inserir em políticas públicas sociais e de saúde, voltadas para suas fragilidades, evitando assim possibilidades de exclusão social e negligência. Deste modo, destacase a importância de se investigar e elencar as ações desenvolvidas pelo NASF Núcleo de Apoio a Saúde da Família com o intuito de ampliar e qualificar a atenção à Saúde do Idoso, frente às especificidades que envolvem o processo de priorizar o atendimento nesse ciclo de vida, pois entendemos que os idosos também se constituem em um grupo heterogêneo, com múltiplas necessidades, de serviços públicos e atenção a esta população, via de acesso e cuidado qualificado no SUS.

Foi possível constatar que 0 atendimento psicossocial constitui-se como uma importante ferramenta na atenção básica para a promoção de saúde e prevenção de agravos em todos os ciclos de vida, incluindo o idoso, levando em consideração que grande parte da população brasileira encontra-se em idade avançada e muitos serviços de promoção à saúde para com essa clientela são negligenciados, ocasionando o agravo de sua condicionalidade bem como excluindo-os do direito preconizado em Lei do atendimento prioritário a saúde.

Ante ao exposto, faz-se primordial refletir, investigar e problematizar sobre todos os aspectos que envolvem o processo de contemplação de atendimento à saúde do idoso, tendo em vista que por intermédio deste se busca oportunizar ao idoso o respeito e cumprimento do amparo legal específico para alguém que tem direitos garantidos e assim ter contemplada sua saúde e integridade física e também psicossocial. A pessoa idosa apresenta profundas particularidades via processo de envelhecimento, e que sua dignidade frente a esse processo não seja negligenciada. 


\section{REFERÊNCIAS}

BRASIL. Constituição da República Federativa do Brasil. 5 de outubro de 1988. Brasília, DF, 1988.

- Ministério da Saúde. Diretrizes do NASF: Núcleo de Apoio Saúde da Família. Cadernos de Atenção Básica, n. 27. Brasília, DF, 2010.

. Presidência da República Casa Civil. Subchefia para Assuntos Jurídicos. Lei n. 10.741, de $1^{\circ}$ de outubro de 2003. (Estatuto do Idoso). Disponível em: http://www.planalto.gov.br/ccivil_03/leis/2003/110.741.htm. Acesso em: 02/05/2018.

. Presidência da República Casa Civil. Subchefia para Assuntos Jurídicos.

Lei n. 8.842, de $4^{\circ}$ de janeiro de 1994. Disponível em: http://www.planalto.gov.br/ccivil_94/leis/1994/.741.htm. Acesso em: 17/05/2018.

CAMACHO, A.C.L.F. Políticas públicas para a saúde do idoso: revisão sistemática. Revista Brasileira de Enfermagem: 2010.

CALDAS, C.P. A saúde do idoso: Arte de cuidar. Rio de Janeiro: Nova Fronteira, 2011.

FILHO, C.B A história da Saúde Pública no Brasil. São Paulo: Ática, 2008.

MORAES, E.M. Atenção à saúde do idoso: Aspectos conceituais. Brasília: Organização Pan-Americana da Saúde; 2012.

VASCONCELOS .M. A priorização da família nas políticas de saúde: Saúde em Debate. São Paulo: Hucitec,2012.

VERAS, R. P. Modelos de atenção à saúde do idoso: Repensando o sentido da prevenção. Revista Brasileira de Geriatria e Gerontologia 2001.

Enviado: Outubro, 2019.

Aprovado: Dezembro, 2019. 